SCIENTIFIC LETTER

\title{
Site of successful slow pathway ablation relates to clinical tachycardia rate in patients with atrioventricular nodal re- entrant tachycardia
}

\author{
N Dagres, A S Manolis, T Maounis, G Poulos, D V Cokkinos, M Borggrefe
}

Heart 2006;92:115-116. doi: 10.1136/hrt.2004.054056

$\mathrm{S}$ low pathway ablation provides curative treatment for patients with atrioventricular nodal re-entrant tachycardia (AVNRT). The major concern remains the induction of complete atrioventricular (AV) block. ${ }^{1}$ To minimise the risk, many centres apply a stepwise approach, targeting initially inferoposterior sites of the septal annulus of the tricuspid valve. ${ }^{2}$ If the first ablation attempts are unsuccessful, the catheter is positioned at more medial and finally anterosuperior sites. Thus, the longest possible distance from the AV node is kept during energy delivery.

Previous investigations suggest that the successful ablation site may be related to the conduction interval of the slow pathway. ${ }^{3-5}$ Since this interval is a determinant of the tachycardia cycle length, we hypothesised that the successful ablation site may be related to the heart rate during tachycardia. Such a finding would be important, since it would possibly allow a rough prediction of the successful ablation site and, thus, an approximate stratification of the risk of AV block based on ECG documentation of the tachycardia.

\section{METHODS}

We studied 109 patients (44 men, 65 women, mean (SD) age 47 (16) years) with AVNRT undergoing slow pathway ablation.

After providing informed written consent, patients underwent the procedures in the fasting, non-sedated state. All antiarrhythmic agents had been discontinued for at least five drug elimination half lives.

The stepwise approach was applied as follows. The septal annulus of the tricuspid valve, extending from the most inferior area of the annulus adjacent to the coronary sinus ostium to the His bundle recording site, was divided into three areas: inferior, medial, and superior. ${ }^{2}$ The His bundle recording site was defined as the most inferior site with a detectable His bundle recording. The ostium of the coronary sinus was determined by the position of a coronary sinus catheter. For slow pathway ablation, the ablation catheter was initially positioned in the inferior, then in the medial, and finally in the superior area, if necessary. After each radiofrequency application, programmed stimulation was applied to assess the presence of slow pathway conduction and inducibility of AVNRT.

To assess the usefulness of tachycardia rate with regard to risk stratification before ablation, we used the heart rate during clinical tachycardia episodes for analysis. Clinical tachycardia was not documented by ECG for 22 of the 109 patients; for these patients, we used the heart rate of tachycardia induced in the electrophysiological study under baseline conditions-that is, in the drug-free state, without administration of isoprenaline or other drugs and without sedation.

Data are presented as mean (SD) and range. Dichotomous variables were compared by the $\chi^{2}$ test. Heart rates during tachycardia episodes in the three groups were compared by one way analysis of variance, followed by the StudentNewman-Keuls test for multiple comparisons. Differences with $\mathrm{p}<0.05$ were considered significant. Data were analysed with the software package SigmaStat version 2.0 (SPSS Inc, Chicago, Illinois, USA).

\section{RESULTS}

The ablation procedure was successful in 107 patients $(98.2 \%)$. One patient $(0.9 \%)$ developed AV block necessitating pacemaker implantation. In this case, the ablation catheter was placed at a medial site.

The successful ablation site was located in the inferior region in 38 patients (35.5\%, group I), in the medial in 54 patients $(50.5 \%$, group $\mathrm{M})$, and in the superior region in 15 ( $14.0 \%$, group $\mathrm{S})$.

The average heart rate during tachycardia increased progressively from group $I$ to group $M$ to group $S$ : 179 (28) beats/min (range 133-260) in group I versus 193 (30) beats/min (range 146-270) in group $M$ versus 212 (38) beats/min (range 150-280) in group $S(p=0.002$, analysis of variance). All pairwise comparisons between the three groups showed a significant difference (fig l).

The proportion of patients with a tachycardia rate $\geqslant 200$ beats/min was higher in group S ( 11 of $15(73.3 \%)$ patients) than in groups $\mathrm{M}$ and I (31 of $92(33.7 \%)$ patients, $\mathrm{p}=0.009)$. A tachycardia rate $<200$ beats $/ \mathrm{min}$ had a negative predictive value of 0.94 for ablation in the superior region. A tachycardia rate $\geqslant 200$ beats/min had a positive predictive value of 0.26 for ablation in the superior region.

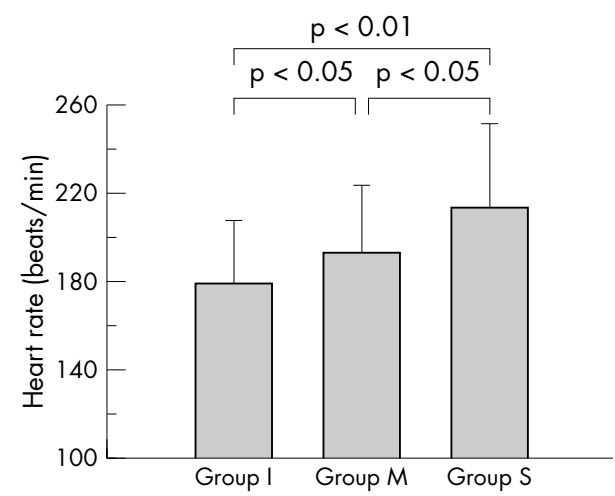

Figure 1 Heart rate during tachycardia episodes in the three groups. Bars depict the standard deviation in each group. Tachycardia rate differed significantly between the groups (analysis of variance $p=0.002$ ). Group I, patients with successful ablation site in the inferior region; group $M$, patients with successful ablation site in the medial region; group S, patients with successful ablation site in the superior region. 
Analysis of the heart rate of the tachycardia induced in the electrophysiological study in the drug-free state showed the same relation between tachycardia rate and successful ablation site: 161 (22) beats/min in group I versus 178 (25) beats/min in group M versus 192 (28) beats/min in group $S(p=0.001$, analysis of variance).

\section{DISCUSSION}

The main finding is that the successful ablation site of the slow pathway is dependent on the tachycardia rate. In patients with slower tachycardias, the slow pathway is ablated at more inferior sites with a lower risk of AV block. A tachycardia rate $<200$ beats/min has a high negative predictive value for ablation in the superior region; ablation in this area carries the highest risk of AV block due to its vicinity to the compact AV node. Thus, ECG documentation of the tachycardia may provide significant information regarding the ablation site and risk of AV block development.

Up to now, almost no clinical information has allowed stratification of the procedure associated risk of AV block before ablation. These findings allow for the first time a limited risk stratification based on the easily measurable tachycardia rate. This not only is important as clinical information, it also may influence the way physicians inform patients concerning the procedure related risk, reassuring those with slow tachycardias of an even lower likelihood of the need for pacemaker implantation.

It is widely accepted that the risk of inadvertent AV block substantially increases with proximity of the target site to the AV node. Nevertheless, ablation in the medial or even inferior area does not exclude the possibility of AV block. ${ }^{1}$

Our findings are consistent with previous studies showing a greater distance between the successful ablation site and the His bundle recording in patients with longer AH intervals during slow pathway conduction. ${ }^{3-5}$

Autonomic tone influences tachycardia rate. To assess the usefulness of tachycardia rate with regard to risk stratification before ablation, we analysed the heart rate during clinical tachycardia episodes. Analysis of the heart rate of the tachycardia induced during the electrophysiological study in the drug-free state confirmed these results, showing the same relation between tachycardia rate and ablation site.

The successful ablation site of the slow pathway depends on the tachycardia rate. In patients with slower tachycardias, the slow pathway is ablated at more inferior sites with a lower risk of AV block. A tachycardia rate $<200$ beats/min has a high negative predictive value for ablation in the superior region. ECG documentation of the tachycardia may provide significant information regarding the ablation site and risk of procedure associated AV block.

\section{Authors' affiliations}

N Dagres*, A S Manolist, T Maounis, G Poulos, D V Cokkinos, 1st Cardiology Department, Onassis Cardiac Surgery Centre, Athens, Greece

M Borggrefe, Department of Cardiology, Angiology and Pneumology, University Hospital of Mannheim, Clinical Faculty of the University of Heidelberg, Mannheim, Germany

*Also the 2nd University Cardiology Department, University of Athens, Attikon University Hospital, Athens, Greece

†Also the 1st Cardiology Department, Evangelismos Hospital, Athens, Greece

There are no competing interests. There are no financial or other associations that might pose a conflict of interest

Presented in part at the European Society of Cardiology Congress in August 2004 in Munich, Germany.

Correspondence to: Dr Nikolaos Dagres, Second University Cardiology Department, Attikon University Hospital, Rimini 1, Haidari, 12462

Athens, Greece; nikolaosdagres@yahoo.de

Accepted 12 May 2005

\section{REFERENCES}

1 Calkins $\mathrm{H}$, Yong $\mathrm{P}$, Miller JM, et al. Catheter ablation of accessory pathways, atrioventricular nodal reentrant tachycardia, and the atrioventricular junction: final results of a prospective, multicenter clinical trial. Circulation 1999:99:262-70

2 Jazayeri MR, Hempe SL, Sra JS, et al. Selective transcatheter ablation of the fast and slow pathways using radiofrequency energy in patients with atrioventricular nodal reentrant tachycardia. Circulation 1992:85:1318-28.

3 Tai CT, Chen SA, Chiang CE, et al. Multiple anterograde atrioventricular node pathways in patients with atrioventricular node reentrant tachycardia. J Am Coll Cardiol 1996;28:725-31.

4 Geller JC, Biblo LA, Carlson MD. Relation between the AH interval and the ablation site in patients with atrioventricular nodal reentrant tachycardia. Pacing Clin Electrophysiol 2004;27:1347-54.

5 Philippon F, Plumb VJ, Epstein AE, et al. Multiple slow AV nodal pathways in individuals with AV nodal reentry: effect of conduction interval on site of pathway ablation [abstract]. Circulation 1993;88(suppl I):I492. 